\title{
3D Nanostructures Grown via Focused Helium Ion Beam Induced Deposition
}

Matthew J. Burch ${ }^{1}$, Anton Ievlev ${ }^{1}$, Songkil Kim ${ }^{1}$, Alex Belianinov ${ }^{1,2}$, Olga S. Ovchinnikova ${ }^{1,2}$, Michael G. Stanford ${ }^{3}$, Kyle Mahady ${ }^{3}$, Brett B. Lewis ${ }^{3}$, Jason D. Fowlkes ${ }^{1,3}$ and Philip D. Rack ${ }^{1,3}$

1. Center for Nanophase Materials Science, Oak Ridge National Laboratory, Oak Ridge, TN.

2. Institute for Functional Imaging of Materials, Oak Ridge National Laboratory, Oak Ridge, TN.

3. Department of Materials Science and Engineering, University of Tennessee, Knoxville, TN.

The next generation of advanced computing devices will require complex nanoscale frameworks that extend into 3D. Focused ion beam induced deposition (FIBID), uses an organometallic precursor gas in a field ion microscope to fabricate nanoscale structures with high-precision, and smaller critical dimensions than current offerings by the focused electron induced deposition (FEBID), or traditional liquid metal source FIBID. [1-4] In this work, we explore the mechanisms behind the competition of material deposition and sputtering during the 3D FIBID process in a Helium Ion Microscope (HIM), via controlled experiments and Monte Carlo simulations. [5] We provide a diagrammatic road-map for synthesis of 3D structures with highly specified geometries, as well as discuss the interplay between key parameters that affect the final product.

The benefits of FIBID with helium ion microscopy are in the small feature size, as well as the ability to grow 3D structures on non-conductive substrates, such as $\mathrm{SiO}_{2}$ for semiconductor manufacturing or polymer substrates for flexible electronics, Figure 1(a,b). Since helium ions are positive, charge compensation can be easily afforded by using a generic electron flood gun. [6] This opens a possibility to new applications such as direct 3D nanoprinting on organic photovoltaics to organic dielectrics.

We demonstrate complex 3D geometric shapes, with features as small as $16 \mathrm{~nm}$, made in a HIM with a gas flow injection system. We rely on automated methods to control the microscope and the gas injection system settings to yield 3D nanostructures as large as $300 \mathrm{~nm}$ made of interconnected parts. Furthermore, we explore the dichotomy between deposition and milling, that occurs as matter interacts with an accelerated ion beam in FIBID of free-standing structures by mapping deposition and milling regimes as a function of beam pitch and dwell time. Simultaneous control of both milling and deposition offers the potential for higher fidelity and purity structures, and the ability to repair structures in-situ for full workflow cycles [7].

References:

[1] Lipp, S. et al, Microelectronics Reliability 36 (1996), p. 1779.

[2] Utke, I., Moshkalev, S., Russell, P., "Nanofabrication using focused ion and electron beams: principles and applications." (Oxford University Press, 2012).

[3] Van Dorp, W., Hagen, C. W., Journal of Applied Physics 104 (2008), p. 10.

[4] Fowlkes, J. D. et al, ACS nano 10 (2016), p. 6163.

[5] Belianinov, A. et al, MRS Bulletin 42 (2017), p. 660.

[6] Hlawacek, G. and Gölzhäuser, A. eds. "Helium Ion Microscopy.” (Cham: Springer International Publishing, 2016).

[7]. The HIM imaging, image analytics and simulations portion of this research was conducted at the Center for Nanophase Materials Sciences, which is a DOE Office of Science User Facility. This research 
was funded by the Center for Nanophase Materials Sciences, which is a U. S. Department of Energy Office of Science User Facility.
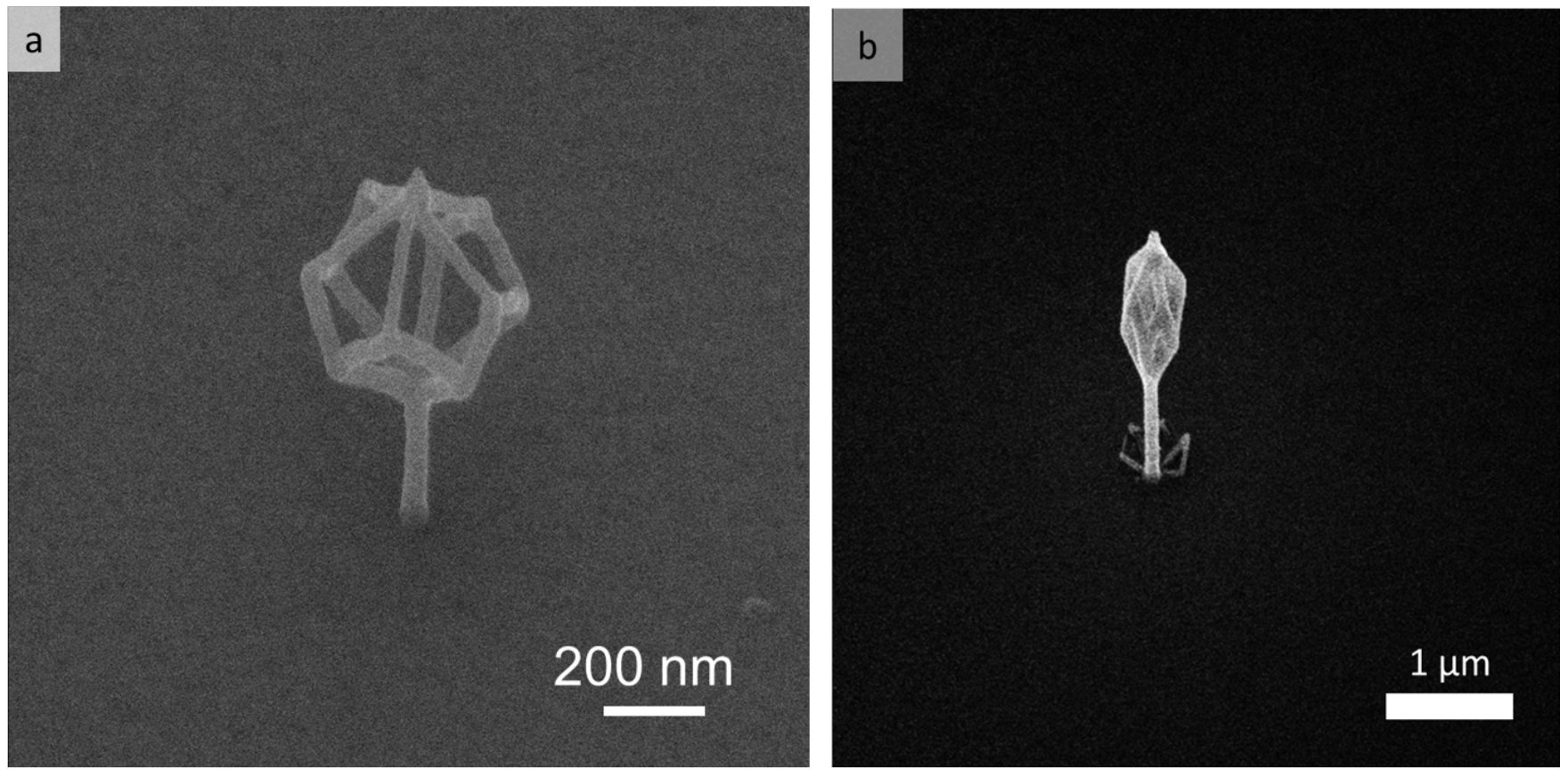

Figure 1. Examples of Helium Ion Microscopy Focused Ion Beam Deposition results using a $\mathrm{MeCpPtMe}_{3}$ precursor. (a) deltahedron grown on a pillar atop a Si substrate and (b) deltahedron grown on a pillar atop $\mathrm{SiO}_{2}$ 